

\title{
LA EVALUACIÓN PROFESORAL DESDE UN CONTEXTO DISCIPLINAR
}

\section{Proffesorial evaluation from a disciplinary context}

Fecha de recepción: 28 de mayo de 2014 - Fecha de aprobación: 1 de diciembre de 2014

\section{RESUMEN}

El presente artículo muestra los resultados de un trabajo realizado con los profesores del Programa Académico de Terapia Ocupacional de la Escuela de Rehabilitación Humana, Facultad de Salud, Universidad del Valle, Colombia en el 2012. El propósito de dicho trabajo fue conocer la percepción que los profesores de esta disciplina tienen frente a la evaluación profesoral y de esta manera conocer necesidades específicas que ayuden a reflexionar sobre el proceso evaluativo actual. Se adelantó un estudio de tipo cualitativo, en donde se utilizó la técnica proyectiva a través de grupo focal. Se trabajó con el $85 \%$ de los profesores nombrados y el $80 \%$ de los profesores contratistas, quienes fueron provocados a la discusión a través de diferentes preguntas que los llevó a puntos de encuentro y desencuentro con relación a su papel y su talante frente a la evaluación profesoral. El profesor universitario debe ser el agente principal de los procesos evaluativos, a través del empoderamiento de dicho proceso, para lograr un sistema óptimo que impacte en el currículo.

PALABRAS CLAVE

Evaluación formativa, personal académico docente, universidad.

\begin{abstract}
ABSTRAC
This paper presents the results of a study conducted with faculty Academic Program Occupational Therapy, School of Human Rehabilitation, Faculty of Health, University the Valle, in 2012. The purpose of this work was to know the perception that teachers of this discipline are facing the teacher assessment and thus meet specific needs to help reflect on the current evaluation process. Qualitative study, where the projective technique was used by focus group was made. We worked with $85 \%$ of those appointed teachers and $80 \%$ of contractors teachers, who were brought to the discussion through different questions that led to points and misunderstanding regarding its role and mood against Teacher assessment. The university teacher should be the main agent of the evaluation processes, through empowerment of the process, to achieve optimal system that impact the curriculum.
\end{abstract}

KEY WORDS

Formative evaluation, academic teaching personnel, universities 
De acuerdo a los referentes teóricos analizados y a los trabajos de investigación nacionales e internacionales revisados, se considera que hay unas claras tendencias con relación con la evaluación del profesor las cuales están enfocadas principalmente en: instrumentos de evaluación profesoral, calidad y equidad, percepción del profesor frente al proceso evaluativo, objetivos de formación, relación de la evaluación profesoral frente al rendimiento de los estudiantes, creación de un perfil de profesor y nuevas propuestas de procesos evaluativos.

La evaluación del profesor de Terapia Ocupacional debe ir más allá, de ser solamente un instrumento de medición y control, pues debe tener fines formativos y de mejora del trabajo profesoral, y así lograr la calidad educativa.

De igual manera, demanda diversos retos, entre los cuales están los relacionados con fomentar el mejoramiento continuo de programas académicos y de las instituciones, a través de la efectividad de los procesos de planeación y de gestión que llevan a una dinamización permanente de aspectos formativos (Salazar, Pool, y Durán, 2014).

Otro punto de vista que se debe considerar en la evaluación del profesor, se relaciona con la promoción de una cultura lejana a orientar y a mejorar los procesos de enseñanza, puesto que se ha centrado en el control y sanción y se ha privilegiado el enfoque instrumentalista.

Con relación a la evaluación del profesor, siguen existiendo vacíos conceptuales, dichos vacíos originan las siguientes preguntas: ¿Qué tanto los docentes admiten cambios en su práctica pedagógica, por razones de su evaluación docente? ¿Cuál es la importancia que los profesores refieren frente a la evaluación docente? ¿Qué tanto la evaluación del profesor ha afectado la práctica pedagógica? ¿Cómo se han modificado las prácticas pedagógicas a partir de la evaluación del profesor? ¿A qué se debe la resistencia a los procesos evaluativos? ¿Cuál es el sentido que los profesores universitarios le otorgan a la evaluación profesoral?¿Desde Terapia Ocupacional, cuáles son los avances que se han realizado con relación a evaluación profesoral?. Se debe tener cuidado con los instrumentos aplicados, pues es diferente la práctica pedagógica a la práctica laboral.

Lo que pretende el trabajo de investigación, es poder conocer la percepción que los profesores del Programa Académico de Terapia Ocupacional de la Universidad del Valle tienen frente a la evaluación profesoral. Con el fin de identificar necesidades específicas, de los profesores de la disciplina, que ayuden a reflexionar sobre proceso evaluativo actual.

\section{MÉTODO}

De acuerdo con el propósito del trabajo, se implementó un método cualitativo en donde se hizo uso de la técnica proyectiva de grupo focal.

Con el grupo focal se consigue información a profundidad con relación a la opinión de las personas, se explora el porqué y el cómo de sus opiniones. El objetivo es trabajar con la información suministrada a través de la discusión que se da al interior del grupo. El dato

El tema de la evaluación profesoral universitaria, es algo que se ha discutido en las Instituciones de Educación Superior y que ha generado investigaciones y escritos con relación al tema. a analizar, es el lenguaje. 
El grupo focal según Onwuegbuzie y cols (2011), es una actividad dirigida por un moderador que emplea una guía, la cual lleva a la discusión a un grupo específico dentro de un enfoque determinado, en donde tiene el control del mismo. Dicha guía se orienta por los objetivos del estudio e incluye preguntas que permiten la discusión abierta.

La dinámica del grupo focal, consiste en realizar una reunión, en donde se llevan a cabo entrevistas grupales abiertas y estructuradas, la cual busca que los participantes discutan y elaboren desde su propia experiencia, elementos relacionados con el objeto de investigación.

La técnica que se utiliza para la investigación, es una reunión en donde se maneja la entrevista grupal estructurada, que busca la discusión de experiencias personales, de temáticas o de hechos sociales, objetos de la investigación.

Con esta técnica se busca conocer opiniones, lo interesante del grupo es que provoca reflexiones y controversias en la discusión. El tema a tratar en la investigación, no es delicado, como para que los participantes se nieguen a contestar o den respuestas neutras.

El tipo de entrevista seleccionada para esta investigación fue la entrevista grupal, en donde se trabajó con base en preguntas concretas y orientadoras, que permitieron ampliarse en el momento que se requirió, las cuales llevaron a la discusión, en donde se partió de lo general a lo específico, de lo fácil a lo difícil y de lo positivo a lo negativo.

Las preguntas elaboradas evocaron el pasado, invitaron a la participación a través de la reflexión, fueron dirigidas de acuerdo al problema de la investigación y se indagó sobre puntos clave que llevaran al cumplimiento de objetivos.

Huerta (2014) considera el grupo focal como una herramienta útil para planificar programas y evaluarlos. Lo fundamental de este trabajo, es que permite la libre expresión frente a aspectos específicos de la investigación, así como facilita la participación de la comunidad académica en los diversos procesos formativos.

Las personas que participaron en el grupo focal, fueron seis profesoras nombradas y siete profesoras contratistas del Programa Académico de Terapia Ocupacional de la Universidad del Valle. Son ocho profesoras nombradas, una de ellas hace la investigación y las 10 profesoras contratistas fueron citadas por la directora del mismo, se excluyeron aquellas que por diversas circunstancias no pudieron asistir y por lo tanto no participaron en la discusión.

Los principios éticos son parte fundamental de la investigación, debido a que representan las normas de comportamiento correcto, justo y respetuoso frente al medio y las personas con las que se está trabajando (Trujillo, 2002).

Los principios éticos en las tareas que se llevan a cabo, no se relacionan solo con la voluntad de actuar de manera correcta, sino que "implica un código compartido, métodos de decisión, criterios de selección y capacitación del personal, formas abiertas de comunicación, una cultura democrática y políticas que reflejen el compromiso social de la organización en su contexto" (Rincón, 2014, p. 313).
En el trabajo se respetaron las opiniones de las profesoras de Terapia Ocupacional que participaron dentro de la misma y de igual manera se tuvo en cuenta que ellas pudieran cambiar sus respuestas, lo cual se consideró y permitió, cuando fue necesario.

Se ha respetado en todo momento la confidencialidad de la información obtenida por parte de las profesoras del Programa Académico en estudio. Así como la privacidad de aquellas que participaron en la investigación.

Al tener en cuenta la responsabilidad social de la investigación, se llevarán a cabo socializaciones a otras personas interesadas en el tema, de los resultados a través de conversatorios y artículos publicados (Aluja, 2004). En primera instancia se considerará compartir la información con la Escuela de Rehabilitación Humana y la Facultad de Salud de la Universidad del Valle.

\section{RESULTADOS}

En el trabajo realizado con los profesores, en donde hubo una interacción directa con ellos, se pudo conocer la percepción que tienen frente a la evaluación que se lleva a cabo dentro de la Universidad.

Para su ejecución, fue de mucha importancia compartir las experiencias vividas por ellas a través de su práctica docente, así como confrontarlas con diferentes situaciones relacionadas con el tema.

Para iniciar, se utiliza como elemento provocador, una gráfica (ver figura 1), que incluye tres formatos de uso institucional.

A manera de lluvia de ideas, se les solicita a las profesoras opinar sobre qué viene a su pensamiento cuando ven la gráfica, las cuales reaccionan con frases como: "asignación académica", "evaluación docente", "actividades académicas administrativas", "métodos de recolección", "sistematización", "estrategias para asignar ciertas funciones y calificar otras", "desempeño docente", "responsabilidad en asignación docente", "mi responsabilidad docente frente a lo que estoy contratado para hacer", "la evaluación que eso genera es responsabilidad mía". Siendo esto el inicio de un enriquecedor trabajo.

\section{Evaluación profesoral y actitud de aprendizaje}

Algunas profesoras consideran la evaluación profesoral como un elemento importante, porque es ahí en donde se logra compartir el aprendizaje con los estudiantes, y a partir de ahí se moldean comportamientos relacionados con el área del conocimiento. Pero resaltan, que dicha actividad no se realiza sólo hacia el quehacer del profesor, o hacia la forma como se transmite el conocimiento, sino que es algo que está mediado por las interacciones sociales y las experiencias. Pero esta mirada, no puede estar puesta sólo en el desempeño docente, sino en la manera en que dicho desempeño, logra impactar en la asignatura de la cual hacen parte los estudiantes.

Así mismo, otras profesoras consideran, que se debe tener en cuenta, que el tiempo de experticia en la docencia, hace que se maduren procesos y que la evaluación es un medio que ayuda a facilitar dicha madurez, coinciden en afirmar que han logrado desarrollo y creci-

REV. COL. REH 2014 || Volumen 13 || Páginas 44 - 53 || 


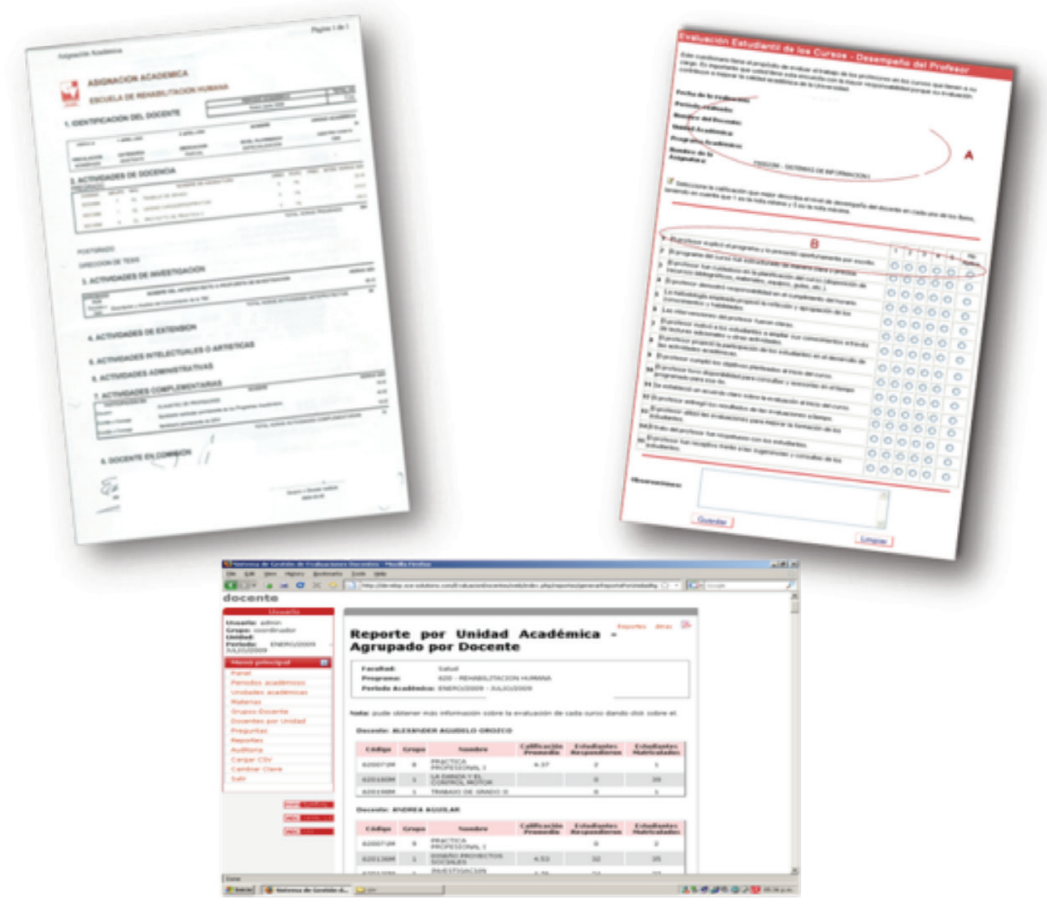

Figura 1. Asignación Académica, Evaluación y Consolidado de la Evaluación Profesoral - Universidad del Valle

miento desde lo pedagógico gracias a los procesos evaluativos, porque han sido éstos procesos, los que les han ayudado a identificar lo que deben mejorar, mantener o potenciar dependiendo de los resultados.

De acuerdo con la opinión de algunas profesoras de Terapia Ocupacional, el proceso educativo está conformado por un sistema que integra asignaturas, currículo, estudiantes, profesores, los cuales deben considerarse en el momento de la evaluación. Ellas perciben que deben estar inmersas dentro del proceso evaluativo, para logar identificar exactamente cuáles son los estándares que se manejan con relación a las asignaturas, las estrategias pedagógicas y todo lo que enmarca el proceso de enseñanza - aprendizaje.

Por otro lado, algunas profesoras del programa académico participante en la investigación, resaltan la importancia, de la participación de diferentes actores de la comunidad académica en los procesos evaluativos, consideran que siendo esta actividad de tal talante, no es conveniente dejarla solo bajo la responsabilidad de los estudiantes, igualmente porque el profesor no solo realiza actividades propias de docencia, sino que tiene a su cargo otras funciones sustantivas.

La evaluación se considera como algo pertinente, puesto que de sus resultados surgen planes de mejoramiento, estrategias y metodologías para que el profesor implemente, lo cual se verá reflejado en términos de calidad.

Reconoce este grupo de profesoras que la evaluación como tal, en un principio es difícil asumirla, pero que una vez conocen e identifican cual es el objetivo con el cual se va a realizar, es realmente acertada.

\section{Evaluación profesoral y actitud de estímulo}

Refiere un grupo de profesoras que la evaluación debe ser amplia y abarcar todas las funciones sustantivas del profesor (docencia, investigación y proyección social), incluyendo las de orden administrativo que también le conciernen, dentro de la Universidad. No debe limitarse, sólo al acto de enseñar. Plantean este punto como una debilidad del proceso evaluativo, puesto que la evaluación es realizada, solo al "profesor dador de clase", aquella de tipo magistral o la que se desarrolla en la práctica con los estudiantes al realizar sus acciones en educación, salud o trabajo. Pero queda por fuera, lo que se denomina como currículo oculto, es decir otras acciones que se hacen, como planes de trabajo, informes de semestre, elementos que no están explícitos en la asignación académica, y que vale la pena resaltar, lo cual excede el trabajo profesoral. Además, siguiendo la línea de la importancia de la evaluación, es significativo comenzar a considerar otros actores dentro del proceso evaluativo, pues actualmente se toma como única voz válida, la del estudiante.

Sin embargo, existen opiniones de otras profesoras del Programa Académico de estudio, que consideran que el objetivo de la evaluación es medir, lo cual los llevará a comparar el estado de su desempeño docente. Éste debe responder, a un estándar o parámetro esperado, y lo ideal es que dichos parámetros puedan ser visibles y que permitan una caracterización, de tipo cuantitativo o cualitativo de 
todas las acciones docentes. Pero dichos estándares deben finalmente revertirse en los aspectos formativos y de esta manera re alimentar la función profesoral.

Otras profesoras del programa consideran, que se debe crear una verdadera cultura evaluativa, que fomente la realimentación del estudiante hacia el profesor, pues en el momento de realizar la evaluación, el profesor es excluido del aula, el formato es aplicado por un tercero y no permiten que se realice un diálogo entre los actores comprometidos con dicho proceso formativo. Se conoce el resultado obtenido en el semestre siguiente, cuando se han consolidado todos los datos, pero de manera cuantitativa.

Reflexionan algunas profesoras, que la evaluación no tiene un proceso como tal, pues lo que se mide es el resultado final de la asignatura. No hay puntos de comparación, pues solo se valora una vez y esto es al final del semestre. Por lo tanto no se proyecta un plan de mejoramiento a lo largo del período académico. Ahora bien, algunas profesoras participantes de la investigación, hacen un llamado con relación a que la educación está mediada por relaciones de poder, la cual puede ser explícita o no. Y que la responsabilidad de la evaluación, se transfiere al estudiante al final del semestre, cuando de alguna manera ya se sabe el resultado de la asignatura, y que este elemento puede influir en la forma de calificar, porque sigue siendo una tarea subjetiva.

De igual manera, consideran que la evaluación debe convertirse en una cultura, donde el acto evaluativo no sea algo punitivo, ni un elemento sacado de los libros o un instrumento diseñado por extraños, por el contrario debe convertirse en un lenguaje cotidiano, esto permitirá un crecimiento académico.

Con todo este panorama, agregan las profesoras de Terapia Ocupacional, que no es para nada pertinente, amarrar la evaluación profesoral a incentivos y estímulos de tipo salarial o de otra índole, pues esto le hace perder su esencia evaluativa, la cual es de realimentación y mejoramiento.

La evaluación, según algunas profesoras participantes, se ha desvirtuado en la medida que es una exigencia gubernamental y que se ofrecen valores agregados, con relación a los puntos docentes. Una buena evaluación significa, que finalmente se revertirá en salario, y desafortunadamente se le ha dado mayor peso a eso, que al sentido que realmente tiene.

Es por esto, que la Directora del Programa Académico de Terapia Ocupacional, hace un llamado de atención, con relación a que la unidad académica, no tiene injerencia sobre los procesos evaluativos de los profesores, debido a que esto, administrativamente, depende de la Dirección de Escuela, quien es el ente gubernamental de los profesores, pero ella considera, valioso tener participación e injerencia en dichos procesos.

\section{Evaluación profesoral y actitud curricular}

Varias profesoras coinciden en que la evaluación, es un elemento que le debe aportar al currículo del Programa Académico, pues el estar inmersos dentro del acto pedagógico, y darse la relación profesor - estudiante, esto inevitablemente debe permear al currículo, las didácticas, la pedagogía, siendo para su opinión, el mayor impacto.

Algunas profesoras consideran, que a pesar de las falencias, la evaluación es una oportunidad, para hacer cambios curriculares y de otra índole. Es una ocasión propicia, para hacer visible lo que en el transcurrir diario se vuelve rutinario. Además, permite hacer reflexiones concretas, sobre elementos del quehacer profesoral, pero además de la misma evaluación. Lo cual permitirá mejorar procesos, como la misma autoevaluación profesoral, en donde ojalá existan unos parámetros establecidos, que le den homogeneidad a dicha actividad. Otras piensan, que cuando se aborda el tema de evaluación, debe analizarse la responsabilidad asignada al estudiante al momento de diligenciar los formatos, afirman que esto es un punto crucial, debido a que realmente nadie les ha enseñado la manera como se debe evaluar.

Diferentes profesoras participantes, encuentran que hay otro elemento que media en la evaluación profesoral y es el instrumento, pues se considera un elemento técnico, que se rige por unas preguntas que indagan aspectos puntuales y que no dan cabida a todas las acciones realizadas en el espacio académico. Colocan como ejemplo, que en el formato se pregunta si el profesor entrega el programa al inicio del semestre, si el profesor entrega los resultados de las evaluaciones a tiempo, pero en ningún ítem se considera el análisis de ese programa o de esas evaluaciones, lo cual indudablemente le aporta a la academia.

Coinciden las profesoras de Terapia Ocupacional, en que hay algo crítico, en ese momento evaluativo, y es que está mediado por las emociones de rabia, satisfacción, tristeza, alegría y estos pueden hacerse evidentes en el instrumento de evaluación. Otra dificultad evidenciada frente a los procesos evaluativos, nace desde su misma concepción, pues ante esa falta de cultura evaluativa, se tiene la idea que se evalúa es para señalar aspectos negativos, para descalificar, para dar por terminado un contrato.

Así mismo refieren que cuando trabajan con grupos interdisciplinarios de la Escuela de Rehabilitación Humana, se llevan a cabo evaluaciones entre pares, las cuales se hacen de manera cualitativa, pero que esto no se considera dentro de la "calificación docente", pues no está en el sistema. Consideran que la evaluación cualitativa es importante, porque coloca en evidencia y re alimenta, lo que realmente pasó en la asignatura, esto permite hacer cambios, reestructuraciones y tomar decisiones.

Como Terapeutas Ocupacionales al hablar de evaluación, analizan varias profesoras, se hace referencia a evaluación del desempeño docente, que para las profesoras significa la mirada que se le hace y que el mismo profesor hace, a su actuar, desde las diferentes funciones en las cuales está inmersa, y con la cuales se ha comprometido a realizar dentro de la institución. A partir de esa mirada y de la mirada de otros actores que intervienen, ellas consideran, se hacen planes de mejoramiento en el propio hacer, lo cual debe revertirse en el quehacer profesoral. 
Concuerdan que además, la evaluación profesoral, indica procesos integrales, que deben estar articulados con la misión y la visión de la Universidad del Valle, de la Facultad de Salud, de la Escuela de Rehabilitación Humana y del Programa Académico de Terapia Ocupacional.

Otras profesoras del mismo programa, indican que la evaluación del desempeño docente es un proceso sistemático, dónde hay diferentes variables y actores. Es la manera como el profesor realiza y desarrolla mejoras o cambios en su desempeño, en su rol como profesor y de todas las actividades que debe realizar dentro de la Universidad.

Un punto interesante que se dio en la discusión con las profesoras de Terapia Ocupacional está relacionado con el término "desempeño" porque se le hace un análisis y se concluye que es un término bastante delicado, puesto que está relacionado con lo empresarial, la producción, el mercado, lo instrumental, es ahí en donde se debe tomar cierta distancia. Pues evaluación profesoral se relaciona con lo académico y desempeño con parámetros de calidad empresarial. Por eso se debe tener cuidado al utilizarla y analizar en realidad que es lo que desea considerar del profesor.

\section{DISCUSIÓN}

De acuerdo a los resultados obtenidos en el grupo focal, realizado con las profesoras del Programa Académico de Terapia Ocupacional de la Universidad del Valle, y a la revisión documental sobre el tema de evaluación profesoral, se logra conocer algunos aspectos referentes a su percepción frente a la evaluación profesoral que se realiza actualmente dentro de la Universidad.

En la actualidad, al igual que en muchas universidades colombianas, la evaluación de los profesores es vista como una acción sumatoria que suele tener en cuenta como principal criterio los resultados de "desempeño docente", valorado por los estudiantes, que son comparados con algunos logros institucionales que se desean obtener.

El actual proceso de evaluación profesoral, tiene un énfasis técnico de corte instrumental, que no sólo es insuficiente para mejorar el desempeño, sino que además no considera el proceso formativo del profesor de Terapia Ocupacional, su aspecto personal, ni su impacto en la formación de los estudiantes. Es importante considerar que la educación es un fenómeno de la condición humana, y por lo tanto está dentro de su faceta humana, de su pensar y repensar (García y Martín, 2013). A la evaluación del profesor le conviene tener en cuenta tanto al educador en su aspecto personal como en sus aspectos profesional y educativo, es decir, ser un proceso integral, el cual requiere ser abordado de manera formativa, y para ello correspondería entablar una relación entre la práctica pedagógica, la evaluación y la formación del profesor.

Rizo (2013) considera que es necesario tener una perspectiva de la evaluación que vaya más allá de lo descriptivo del quehacer profesor, es decir que debe posibilitar sus habilidades y que tener como meta el mejoramiento continuo. Lo cual no se logra, si no se rompen paradigmas con énfasis tecnicista y de carácter positivista.
Entre las estrategias de la evaluación profesoral, existen unas de índole institucional, que se preocupan por la calidad de la educación ofrecida, por eso la aspiración es que la evaluación profesoral, sirva como proceso formativo y guía, que actúe como herramienta fundamental en el logro de la excelencia educativa. Desde esta percepción de calidad, se deben seguir unos elementos establecidos por la Ley General de la Educación, en donde la formación de los profesores universitarios debe cumplir con unos requisitos mínimos de calidad, para lo cual aporta en gran medida los procesos de acreditación institucional en Colombia, los cuales certifican ante la sociedad lo referente con la calidad y la credibilidad en la Universidad, sus docentes, sus procesos formativos, entre otros.

Para el Estado, es fundamental, trabajar con estándares de calidad, los cuales sirven, además, como mecanismos de selección. Bajo estos estándares, establecidos a nivel nacional, se crea dentro de la Universidad del Valle, el estatuto que rige el actuar de los profesores, en donde se determina la dedicación y niveles de formación, el cumplimiento de las funciones universitarias, la interacción con comunidades, la producción escrita y su remuneración, en donde puede tener incentivos (Consejo Nacional de Acreditación, 2013). Pero considerar al profesor de Terapia Ocupacional desde una mirada global generalizada, dentro los procesos de acreditación institucional, hace que se pierda la dimensión del profesor como sujeto.

Si las Universidades en realidad, buscan modificar los programas académicos, las prácticas pedagógicas y didácticas; se debe ver la evaluación profesoral desde su naturaleza científica y no solo desde lo técnico. Por esto es importante que se profesionalice a los encargados de desarrollar, implementar y sistematizar los resultados de dicha evaluación (Montoya, Abersú, Contreras y Serrato, 2014).

El profesor de Terapia Ocupacional como agente reflexivo, analítico, crítico, que lidera procesos sobre la enseñanza, que tiene una perspectiva pedagógica, tiene elementos suficientes para aportar y agenciar elementos propios de su evaluación. Pero es clara la tendencia, con base en diferentes investigaciones de Latinoamérica con relación a evaluación profesoral, que una vez se han hecho las indagaciones respectivas, se llega a la reconstrucción del instrumento, pues de acuerdo a los resultados, se concluye que es éste, quien no ofrece los elementos necesarios para valorar al profesor. Cae nuevamente la evaluación, en una herramienta de medición, no como elemento que agencia y moviliza cambios en la comunidad académica.

La evaluación, no debe dejarse sólo al margen del uso de una técnica o instrumento, pues existen varios elementos que pueden intervenir en el proceso. Como por ejemplo, los propósitos, el reconocimiento de la naturaleza de lo que se somete a evaluación, a quién se le realizan la evaluación y las fuentes de información (Rueda, 2011).

Se ha desdibujado el objetivo de la evaluación profesoral, pues en la actualidad es vista como un elemento de control que ejercen las instituciones de educación, en donde lo que se busca es comparar los niveles logrados de acuerdo a las normas que rigen el quehacer del profesor. Por ser un elemento de control, la responsabilidad y la dinamización de los procesos evaluativos, recaen en personal administrativo, que hacen uso de los dispositivos institucionales, que limitan la participación de los profesores en el proceso (Diego y Rueda, 2012). 
Es por esto, que las instituciones deben hacer acercamientos, hacia una evaluación profesoral diferente, además porque sus profesores, como en el caso de Terapia Ocupacional, de la Universidad del Valle, así lo reclaman, un buen ejemplo es la evaluación sistemática, que se ha considerado tiene que ver con lo pedagógico, en donde se busca especificar los diferentes procedimientos de evaluación, los métodos de enseñanza, las estrategias de aprendizaje, con el fin de interrelacionarlo con lo curricular, la misión de la universidad y el entorno. Dentro de este marco, se retoma lo referente a evaluación formativa, la cual considera a la enseñanza como un proceso que ayuda a tomar decisiones y determina al profesor como un profesional que se encarga de adoptarlas (Stuflebeam, 1993).

La evaluación profesoral, según lo afirmado por Montoya et al (2014) debe admitir lo variada y compleja que es la actividad profesoral, razón por la cual no hay una "receta" ni una serie de técnicas que permita evaluarla de manera sencilla. Los profesores y directivos en cada universidad deben identificar y reflexionar en torno a aspectos que favorecen su tarea docente y como apuntan a un mejoramiento continuo. Además la evaluación profesoral en Terapia Ocupacional debe permitir comprender el ejercicio profesoral, a través de juicios que aporten a la mejora de su praxis.

En este sentido Quintar (2009) ha destacado que los procesos evaluativos, para asumir una dinámica formativa e integral, requieren de espacios pedagógicos en los que el profesor y el alumno descubran nuevas formas de abordar el conocimiento en los que haya una verdadera construcción de sentidos y significados, requiere de un ambiente creativo en el aula. Para lograrlo habría que obtener la confianza entre estos dos sujetos que tradicionalmente se han mirado con aprehensión y desconfianza, lo cual no es fácil y para que surja debe existir un relacionamiento no basado en el rigor lógico sino en la captación de aquello que esencialmente nos vincula y nos une: lo humano, aspecto poco considerado dentro de los procesos evaluativos profesorales. Evidentemente, dicha dimensión humana, tiene el riesgo de restringirse a lo instrumental si se limita a aplicar instrumentos orientados a evaluar la ejecución práctica del quehacer profesoral dentro de los salones de clase.

La autoevaluación es otra mirada que se hace a los procesos evaluativos, en la cual quien evalúa y quien es evaluado son la misma persona. Este es un ideal que parte de la reflexión, análisis y crítica de cada profesor sobre su quehacer, el cual debe revertirse a un mejoramiento continuo. Pues es él quien conoce su actividad e identifica en dónde están sus falencias y por lo tanto cómo mejorarlas.

La autoevaluación como elemento pedagógico según Canney (2014), necesita de un seguimiento permanente, en donde se identifiquen logros y elementos no alcanzados en el proceso formativo, de igual manera las adecuaciones al proceso de enseñanza, si así se requiere. Lo que incluye un seguimiento a los diversos enfoques de aprendizaje, permite conocer estrategias de comprensión, le otorga un sentido al aprendizaje y facilita en la comunidad académica el logro de objetivos dentro del proceso formativo. Este es uno de los elementos que se consideran en los procesos evaluativos en la Educación Superior, pero los esfuerzos hacia una verdadera auto reflexión presenta cierto grado de dificultad, que no ha podido ser superada a través del tiempo. Falta crear una cultura de la misma y concientizar a los profesores de Terapia Ocupacional, de la Universidad del Valle, de la importancia de este proceso evaluativo.

El profesor universitario como sujeto integral, implica al evaluarlo, que se le debe reconocer desde sus aspectos personales, intelectuales, académicos y sociales. Su abordaje exhaustivo demanda identificar su diversidad emocional y mental. En este sentido Damasio (2010) plantea, que las representaciones se construyen en el cerebro humano y describen diferentes situaciones a lo cual responde el cuerpo con movimientos, pero esto depende de las interacciones mente-cuerpo.

Damasio (2010) tiene la hipótesis de que la mente surge de un organismo y no de un cerebro que está separado del cuerpo. Por esto no es objetivo dejar las emociones y los sentimientos fuera de la concepción global de la mente. Los sentimientos tienen influencia sobre la razón, puesto que los sistemas cerebrales están relacionados entre sí y regulan al cuerpo. La emoción entonces, está enmarcada dentro de una acción de impulsos biológicos y estados corporales los cuales son fundamento indispensable para la racionalidad. De igual manera, afirma que el papel de los sentimientos en la construcción de la razón tiene implicaciones para algunos temas a los que la sociedad se enfrenta normalmente, entre ellos la educación y la violencia. Es por esto que el ser humano es complejo, frágil, finito y único. Elementos que no se pueden dejar por fuera en un momento de evaluar a las profesores de Terapia Ocupacional, por el contrario deben de potenciar, el trabajo que se hace ahí.

Para la racionalidad es indispensable la acción de los impulsos biológicos, los estados corporales y la emoción. A su vez los sentimientos como elemento fundamental para el ser humano no se aíslan de su cuerpo ni de sus movimientos. Evaluar al profesor de Terapia Ocupacional, de la Universidad del Valle, implica por tanto abordar las interacciones mente-cuerpo, en otras palabras, sus emociones y capacidades cognitivas.

Los sentimientos en un proceso evaluativo influyen, por un lado para quien la realiza y por otro para quien la recibe, pues los estímulos formativos positivos o negativos así como los resultados, en algunos casos, dependen de la interacción que se haya tenido a lo largo del proceso educativo, y estos aspectos emocionales del aprendizaje influyen en la formación y desarrollo humano tanto de estudiantes como de profesores del Programa Académico de Terapia Ocupacional, en estudio, así como en la actitud, de dicha comunidad académica hacia el conocimiento y hacia la evaluación que requiere ser más valorada y conocida.

Las emociones pueden facilitar o inhibir los procesos educativos. Puesto que son quienes determinan el dominio de acción en que se mueve cada individuo. Son prácticas corporales que determinan o especifican influencias de acciones (Maturana, 2005). En los procesos educativos por ejemplo, el objetivo se centra en crear un mundo con el otro, en donde los evaluadores logran ser la referencia de esa creación; por tanto la evaluación debe observar el hacer y el ser del profesor. La evaluación no debe enjuiciarlo, por el contrario debe darle herramientas que le ayuden a fortalecer su quehacer (Maturana, 2005). 
Lo anterior permite distinguir vacios conceptuales que son importantes de trabajar en investigaciones futuras, con relación al tema de la evaluación profesoral, tal como, la evaluación como proceso, qué tan válido es el incentivo laboral como mediador en la evaluación profesoral, actores que deben participar en la misma, motivación e interés profesoral en su evaluación, participación activa del profesor en la evaluación entre otras.

A pesar que las políticas educativas de los últimos años, en Colombia, se reflexiona sobre la importancia del proceso de evaluación, es importante avanzar e ir mucho más allá de aspectos netamente administrativos y enfocarlas más hacia elementos que fortalezcan y le propicien desarrollo integral al profesor de Terapia Ocupacional, de la Escuela de Rehabilitación Humana, de la Facultad de Salud, de la Universidad del Valle. La evaluación del profesor de Terapia Ocupacional, debe ser precursora de la excelencia formativa y académica dentro del contexto de la Escuela de Rehabilitación Humana, de la Facultad de Salud, de la Universidad del Valle y por lo tanto del sistema Educativo Superior de Colombia. Para lograr que el sistema de evaluación profesoral triunfe, es el profesor de Terapia Ocupacional, de la Universidad del Valle, quien como agente principal, se debe empoderar del mismo, tomar decisiones frente a la manera como se desarrolla, así como del impacto que debe tener.

La evaluación del profesor de Terapia Ocupacional de la Universidad del Valle, sigue un enfoque técnico de corte instrumental, que no sólo es insuficiente para mejorar su práctica pedagógica, sino que además no considera el proceso formativo de ellas, su aspecto personal, ni su impacto en la formación de los estudiantes. Razón por la cual, debe dejar de ser un elemento estático e instrumental, debe ser dinamizador de procesos de mejoramiento en la formación del Programa Académico.

El papel de la evaluación se convierte, en un elemento que supervisa, controla y mide los resultados obtenidos de las profesoras de Terapia Ocupacional, de la Universidad del Valle, en donde lo que finalmente busca es evaluar al individuo, encargado de la educación. No se considera el proceso, se analiza como producto terminado. Frente a los hallazgos se puede decir entonces, que la evaluación del profesor de Terapia Ocupacional de la Universidad del Valle, debe ir más allá de ser solamente un instrumento de medición y control, pues debe tener fines formativos y de mejora del trabajo profesoral, y así lograr la calidad educativa.

Se debe hacer un gran esfuerzo por fortalecer la evaluación pedagógica, para que se fortalezca la unión de lo curricular, lo pedagógico y lo didáctico, el papel del profesor del Programa Académico es fundamental, debe ser líder del proceso. El profesor de Terapia Ocupacional, de la Universidad del Valle, debe asumir un papel protagónico, comprometerse con su evaluación, sentirla como parte de él.

En los últimos años, ha ingresado a la educación el término calidad, el cual no es fácil de asumir y de aplicar. Razón por la cual, se ha creado la necesidad de diseñar un nuevo perfil profesional para el profesor, que lo coloca en un plano productivo no académico. Es responsabilidad del profesor resarcir su función primordial desde lo formativo. Las instituciones de educación superior, ante el ingreso del término calidad, ven en la evaluación una oportunidad para realizar seguimiento y control. Es función de los profesores de Terapia Ocupacional, de la Universidad objeto de estudio, dinamizar y reflexionar en torno al tema evaluativo y no dejarlo como un elemento de control.

El profesor de Terapia Ocupacional ha ido cediendo espacio, ante esta nueva estructura que ha ingresado a la educación, se le profesionaliza más y la universidad trata de ser homologada con una empresa, por esto se han incorporado nuevas categorías tales como, perfil profesional, valor social y de mercado de su trabajo, tipos de contenidos, especializados y formas de adquisición, habilidades profesionales, manejo de nuevas tecnologías, sistemas de acreditación y evaluación. Pero la función pedagógica no puede perderse, ni dejarse de lado, el profesor tiene una responsabilidad frente a la comunidad académica que no puede dejar de cumplir.

La evaluación del profesor de Terapia Ocupacional de la Universidad del Valle, debe ser un diálogo permanente con el contexto del programa académico, para lograr articular lo teórico y lo práctico, para lograr disminuir la brecha entre el saber y el quehacer. Existen unas tendencias conceptuales, de acuerdo con investigaciones realizadas a nivel local e internacional, que dejan al descubierto falencias en los procesos evaluativos, como profesores, nos enfrentamos a un gran reto que es ofrecer a la comunidad académica elementos propios de la educación, que logren impactar en realidad en lo curricular.

Un aspecto que es interesante, según lo referido por Hamodi, López y López (2015), es que la evaluación profesoral es algo que le pertenece a todos, pero de la misma manera los beneficia, se considera como una oportunidad de aprendizaje para todos los agentes comprometidos, en donde lo que se busca es "estimular capacidades y pensamiento crítico - reflexivo, sobre el proceso de aprendizaje, la autonomía y la autorresponsabilidad", por esto es necesario que salga del concepto unidireccional y de medición.

\section{RECOMENDACIONES}

Es recomendable que los profesores de Terapia Ocupacional comiencen a hacerse responsables y comprometidos con el proceso evaluativo, y que sea desde sí mismos que surjan los modelos y las prácticas de evaluación. Las investigaciones que se hagan sobre el tema, deben mirar mucho más allá, que el instrumento, se deben analizar los elementos propios de la educación.

Finalmente, se recomienda que las investigaciones sobre el tema de evaluación profesoral continúen, con el fin que se fortalezca el quehacer profesoral, su práctica, sus didácticas y todo lo relacionado con los procesos formativos, para que finalmente la evaluación deje de ser un elemento instrumental y logre conseguir su objetivo de re alimentar y mejorar los procesos pedagógicos.

\section{AGRADECIMIENTOS}

Se agradece de manera especial a las profesoras del Programa Académico de Terapia Ocupacional de la Universidad del Valle, por haber participado de manera activa y motivada en esta investigación.

A la Escuela de Rehabilitación Humana, la Facultad de Salud y a la Universidad del Valle, en cabeza de sus directivos, quienes me han dado la oportunidad de poder realizar este trabajo. 


\section{REFERENCIAS}

Aluja, M. (2004). Aspectos Éticos en el Uso del Conocimiento. En A. B. Aluja, Aspectos Éticos en el Uso del Conocimiento (págs. 47-51). México: Academia Mexicana de Ciencias.

Canney, M. (2014). La Autoevaluación por el Estudiante: aproximación a las creencias de docentes universitarios. Tesis de Maestría. Pontificia Universidad Javeriana: Bogotá. Recuperado en Marzo 19 de 2015, http://hdl.handle.net/10554/12356.

Consejo Nacional de Acreditación (2013). Lineamientos para la Acreditación de Programas Académicos de Pre Grado. Factores e indicadores de evaluación, CNA, Ministerio de Educación, Bogotá.

Damasio, A. (2010). El Error de Descartes. Barcelona: Drakontos.

Diego, M. \& Rueda M. (2012). La Evaluación Docente en Educación Superior: uso de instrumentos de autoevaluación, planeación y evaluación por pares. Voces y silencios: Revista Latinoamericana de Educación, Vol. III, Núm. 2, 59-76.

García, W. \& Martín, M. (2013). Hermenéutica y Pedagogía. La práctica educativa en el discurso sobre la educación. Revista Educación Pulso. Vol 36. p. 55-78.

Gómez, V. M. (2000). Cuatro Temas Críticos de la Educación Superior en Colombia. Debate, ASCÚN, Asociación Colombiana de Universidades, Bogotá.

Hamodi, C., López, M. \& López, A. (2015). Medios, Técnicas e Instrumentos de Evaluación Formativa y Compartida en Educación Superior. Revista Perfiles Educativos. Vol. XXXVII. No. 147. IISUE-UNAM: México. p146-161.

Huerta, J. M. (2014).Los grupos focales. Recuperado en marzo 18 de 2015: http://academic.uprm.edu/jhuerta/HTMLobj - 94/Grupo_Focal.pdf

Maturana, H. (2005). Emociones y Lenguaje. En H. Maturana, Emociones y Lenguaje (págs. 14-18). Chile: J.S. Sáez.

Montoya, J., Abersú, I., Contreras, G. \& Serrato, S. (2014). Evaluación de la docencia universitaria en México, Chile y Colombia: Análisis de

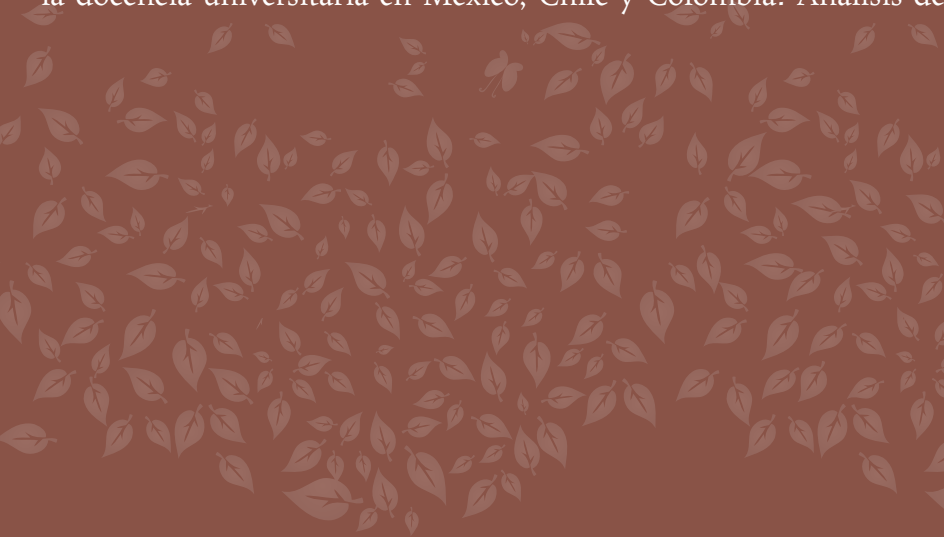

experiencias. Revista Iberoamericana de Evaluación Educativa. Vol. 7. No. 2 (especial). P15-42.

Onwuegbuzie, A., Dickinson, W., Leech, N. \& Zoran, A. (2011). Un Marco Cualitativo para la Recolección y Análisis de Datos en la Investigación Basada en Grupos Focales, 3 (2), 127-157.

Quintar, E. (2009). La Enseñanza como Puente de Vida. En E. Quintar, La Enseñanza como Puente de Vida (pág. 56). México: Instituto Politécnico Nacional. Colección Conversaciones Didácticas.

Rincón, I. (2014). Inteligencia Ética y Calidad de Servicio en las Instituciones de Educación Superior. Revista en línea ENTELEQUIA. No. 17. www.eumed.net/entelequia.

Rizo, H. (2013). Evaluación del Docente Universitario. Una visión institucional. Recuperado marzo 18 de 2015: www.rieoei.org/deloslectores/883Rizo.pdf.

Rodríguez, J. G. (2008). Evaluación del Profesorado en las Universidades Públicas: una aproximación a la situación de Colombia. Revista Iberoamericana de Evaluación Educativa (En Línea), 1(3).

Rueda, M. (2013). La Evaluación Educativa: Análisis de sus prácticas. Ediciones D.D.S. México: Distrito Federal.

Rueda, M. (2011). La Evaluación de la Docencia en la Universidad. Perspectivas desde la investigación y la intervención profesional (Segunda Edición ed.). México: Plaza y Valdez S.A.

Salazar, A., Pool, W. \& Durán, A. (2014). Evaluación Educativa: en la mejora continua de la educación. SEGEY. Secretaría de Educación: Yucatán.

Stuflebeam, D. (1993). Evaluación Sistemática (guía teórica y práctica). Barcelona: Paidós.

Trujillo, A. (2002). Comunidad Profesional. En A. Trujillo, Conocimiento y Práctica en Colombia (pág. 417). Bogotá: Universidad Nacional de Colombia. Sede Bogotá. Colección Sed.

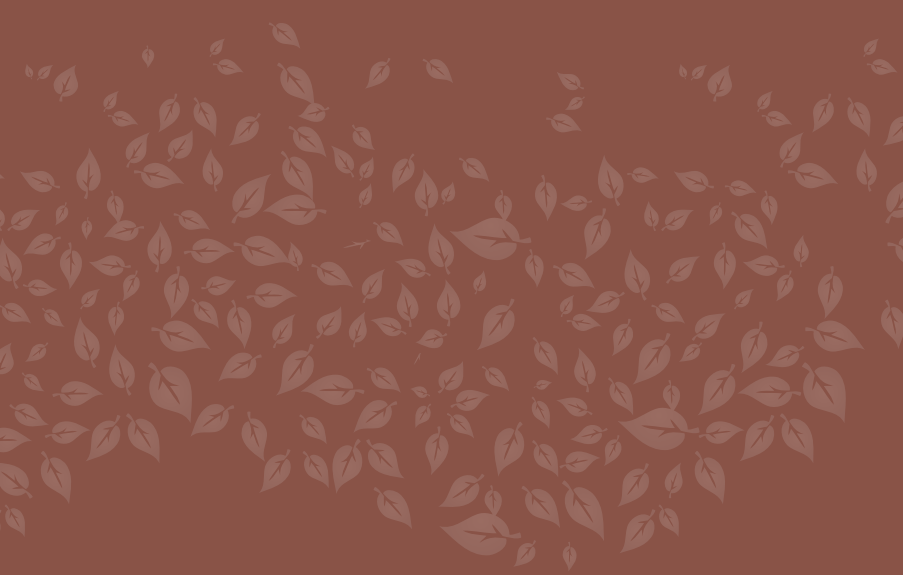

REV. COL. REH 2014 || Volumen 13 || Páginas 44 - 53 || 